\title{
Lagrangian study of transport and mixing in a mesoscale eddy street
}

\author{
S.V. Prants, M.V. Budyansky, V.I. Ponomarev, M.Yu. Uleysky \\ Pacific Oceanological Institute of the Russian Academy of Sciences, \\ 43 Baltiiskaya st., 690041 Vladivostok, Russia
}

\begin{abstract}
We use dynamical systems approach and Lagrangian tools to study surface transport and mixing of water masses in a selected coastal region of the Japan Sea with moving mesoscale eddies associated with the Primorskoye Current. Lagrangian trajectories are computed for a large number of particles in an interpolated velocity field generated by a numerical regional multi-layer eddy-resolving circulation model. We compute finite-time Lyapunov exponents for a comparatively long period of time by the method developed and plot the Lyapunov synoptic map quantifying surface transport and mixing in that region. This map uncovers the striking flow structures along the coast with a mesoscale eddy street and repelling material lines. We propose new Lagrangian diagnostic tools - the time of exit of particles off a selected box, the number of changes of the sign of zonal and meridional velocities - to study transport and mixing by a pair of strongly interacting eddies often visible at sea-surface temperature satellite images in that region. We develop a technique to track evolution of clusters of particles, streaklines and material lines. The Lagrangian tools used allow us to reveal mesoscale eddies and their structure, to track different phases of the coastal flow, to find inhomogeneous character of transport and mixing on mesoscales and submesoscales and to quantify mixing by the values of exit times and the number of times particles wind around the eddy's center.
\end{abstract}

Keywords: Lagrangian transport, mesoscale eddy dynamics, Lyapunov exponents

\section{Introduction}

Large-scale coherent structures, such as major currents and eddies, are key ingredients organizing ocean flows. They are easily seen on surface temperature and sea-surface height satellite data. In numerical models, contours of potential vorticity can be used to reveal coherent structures. However, those and other Eulerian means give snapshots at fixed times and contain little information about transport and mixing of water masses which are inherent Lagrangian notions. Lagrangian approach to study horizontal transport and mixing in the ocean is rapidly becoming an effective method to demonstrate inhomogeneous character of that mixing and existence of coherent structures (Haller and Poie, 1998; Samelson and Wiggins, 2006; Koshel and Prants, 2006; Haller, 2000; Haller and Yuan, 2000;

Email address: prants@poi.dvo.ru (S.V. Prants) URL: www.dynalab.poi.dvo.ru (S.V. Prants)
Shadden et al., 2005; Ide et al., 2002; Mancho et al., 2006; Jones and Winkler, 2002; Samelson, 1992; Artale et al., 1997; Koshel et al., 2008; Izrailsky et al., 2008). There are interesting papers on that approach studying transport and mixing in different basins in the World Ocean with numerically generated velocity fields (Beron-Vera et al., 2008: d'Ovidio et al., 2004; Mancho et al., 2008; Olascoaga et al., 2006; García-Olivares et al., 2007; Miller et al., 1997) and velocity fields derived from satellite altimeter measurements (d'Ovidio et al., 2009; Waugh et al., 2006; Abraham and Bowen, 2002; Lehahn et al., 2007) or using high-frequency radars (Lekien et al., 2005; Lipphardt et al., 2006; Gildor et al., 2009). This approach does not aim at studying individual trajectories of fluid particles but at searching for and identifying spatial structures organizing the whole flow and known in theory of dynamical systems as invariant manifolds.

Motion of a fluid particle is the trajectory of a dynamical system with given initial conditions gov- 
erned by the velocity field computed either by solving the corresponding master equations or as the output of a numerical ocean model or derived from a measurement. The phase space of that dynamical system is the real space where many important phase-space objects known in the theory of dynamical systems, such as stationary points, KolmogorovArnold-Moser tori, stable and unstable manifolds, periodic and chaotic orbits, etc., can be found and studied.

In this work, we use velocity data from the Japan Sea circulation model (Shapiro, 2000) to study and characterize surface transport and mixing in the region comprising the Primorskoye (Liman) Current which is known by its rich mesoscale activity. The instabilities of this current in the warm period generate mesoscale coastal eddies (Ponomarev and Trusenkova, 2000) which propagate downstream until the entrance to the Peter the Great Bay (the latitude of Vladivostok, Russia). These eddies are generated in the model as a kind of a mesoscale eddy street. The mesoscale and submesoscale dynamics over the shelf and steep continental slope includes jet currents, streamers and eddies being controlled by synoptic scale wind forcing and sea baroclinicity. According to satellite data, the anticyclonic mesoscale eddies of relatively small scale of the order $(10 \div 50) \mathrm{km}$ have been observed in the northwestern marginal area directly over the steep continental slope. Near the sea surface they often form pairs of strongly interacting eddies with spiral patterns visible at satellite images. The anticyclonic mesoscale eddies of larger scale have been clearly seen in the southern marginal area of the slope and shelf of the Peter the Great Bay. The mesoscale dynamics over the continental slope could be associated with the coastal Kelvin waves propagating downstream with the Primorskoye Current to the southwest and catched by the wide shelf of the Peter the Great Bay.

Many practical transport problems focus on tracking the evolution of small fluid patches (clusters of particles) near the coast that becomes important in the case of oil and other pollutant spills or harmful algal blooms. It is of crucial interest to know the fate of the particles inside the patch. As to basic research problems in physical oceanography, it is important to know typical transport pathways along which coastal waters move to the open sea and the open-sea waters move to the coast.

The aim of this paper is to study surface trans- port and mixing along the mesoscale eddy street by computing Lagrangian trajectories for a large number of particles advected by the Japan Sea circulation model known as the MHI model (Marine Hydrophysical Institute, Sevastopol, Ukraine) (Shapiro, 2000). Our paper is organized as follows. In section 2, we review briefly the dynamical systems method to study transport and mixing in the ocean. Section 3 introduces the Japan Sea circulation model. Section 4 contains our main results. (i) We compute finite-time Lyapunov exponents (FTLE), the time of exit of a large number of particles off a selected sea region and streaklines. It enables us to reveal mesoscale eddies, hyperbolic and non-hyperbolic regions in the sea and to quantify mixing by values of the FTLE. (ii) We track the evolution of material lines, crossing the eddies, and demonstrate inhomogeneous character of transport and mixing on mesoscales and submesoscales that can be quantified by values of exit times and the number of times particles change their zonal velocity while moving in the preselected box. (iii) We show that the evolution of neighbour patches of particles, chosen on a ridge of largest values of the FTLE field and nearby, illustrates strongly different transport paths of particles in hyperbolic sea regions. We summarize our results in section 5 . In Appendixes $\mathrm{A}$ and $\mathrm{B}$, we illustrate the complex pattern of chaotic mixing and transport by an idealized example of the vortex flow and describe the method of computing the FTLEs, respectively.

\section{Dynamical systems method to study transport and mixing in the ocean}

In Lagrangian approach, a fluid particle is advected by the two-dimensional Eulerian velocity field

$$
\frac{d x}{d t}=u(x, y, t), \quad \frac{d y}{d t}=v(x, y, t),
$$

where $(x, y)$ is the location of the particle, $u$ and $v$ are the zonal and meridional components of its velocity at the location $(x, y)$. The motion is considered on a plane because of a comparatively small size of the region we will analyze in this paper. Even if the velocity field is fully deterministic, the Lagrangian trajectories may be very complicated and practically unpredictable. It means that a distance between two initially nearby particles grows exponentially in time

$$
\|\delta \mathbf{r}(t)\|=\|\delta \mathbf{r}(0)\| e^{\lambda t},
$$


where $\lambda$ is a positive number, known as the Lyapunov exponent, which characterizes asymptotically (at $t \rightarrow \infty)$ the average rate of the particle dispersion, and $\|\cdot\|$ is a norm of the vector $\mathbf{r}=(x, y)$. It immediately follows from (2) that we are unable to forecast the fate of the particles beyond the socalled predictability horizon

$$
T_{p} \simeq \frac{1}{\lambda} \ln \frac{\|\Delta\|}{\|\Delta(0)\|},
$$

where $\|\Delta\|$ is the confidence interval of the particle location and $\|\Delta(0)\|$ is a practically inevitable inaccuracy in specifying the initial location. The deterministic dynamical system (1) with a positive maximal Lyapunov exponent for almost all vectors $\delta \mathbf{r}(0)$ (in the sense of nonzero measure) is called chaotic. It should be stressed that the dependence of the predictability horizon $T_{p}$ on the lack of our knowledge of exact location is logarithmic, i. e., it is much weaker than on the measure of dynamical instability quantified by $\lambda$. Simply speaking, with any reasonable degree of accuracy on specifying initial conditions there is a time interval beyond which the forecast is impossible, and that time may be rather small for chaotic systems.

In the last two decades, dynamical systems methods have been applied to study transport and mixing processes in the ocean (Haller and Poie, 1998; Samelson and Wiggins, 2006; Koshel and Prants, 2006; Haller, 2000; Haller and Yuan, 2000; Shadden et al., 2005; Ide et al., 2002; Mancho et al., 2006; Jones and Winkler, 2002; Miller et al., 1997; Samelson, 1992; Artale et al., 1997). Since the phase plane of the two-dimensional dynamical system (1) is the physical space for fluid particles, many abstract mathematical objects from dynamical systems theory are material surfaces, points and curves in fluid flows. Stagnation point in a steady flow is the fluid particle with zero velocity. Besides "trivial" elliptic stagnation points, the motion around which is stable, there are hyperbolic (saddle) stagnation points which organize fluid motion in their neighbourhood in a specific way. There are two opposite directions (for each saddle point) along which nearby trajectories approach the point at an exponential rate and two other directions along which nearby trajectories move away from it at an exponential rate.

We recall briefly some important notions from dynamical systems theory that will be used in the present paper. Invariant manifold in a twodimensional flow is a material line, i. e., it is composed of the same fluid particles in course of time. To introduce the notion of stable and unstable manifolds it is instructive to consider a steady flow around a hyperbolic point which is the fixed point the fluid motion around which is unstable. So, a fluid particle approaches the hyperbolic point along its stable (unstable) invariant manifolds when $t \rightarrow+(-) \infty$. Even in simple time-periodic flows, the stable and unstable manifolds may intersect each other transversally creating so-called homoclinic and heteroclinic tangles where the fluid motion is so complicated that it may be strictly called chaotic, the phenomenon known as chaotic advection (Aref, 1984; Ottino, 1989; Samelson, 1992; Pierrehumbert and Yang, 1993; Koshel and Prants, 2006; Samelson and Wiggins, 2006). Close fluid particles in the tangles rapidly diverge providing very effective mechanism for mixing. Stable and unstable manifolds are important organizing structures in the flow because they attract and repel fluid particles (not belonging to them) at an exponential rate and partition the flow into regions with different types of motion. Thus, they are transport barriers.

Stable and unstable manifolds are useful tools in studying realistic flows modeling the ocean. In aperiodic flows it is possible to identify aperiodically moving hyperbolic points with stable and unstable effective manifolds (Haller and Poje, 1998). Unlike the manifolds in steady and periodic flows, defined in the infinite time limit, the "effective" manifolds of aperiodic hyperbolic trajectories have a finite lifetime. The point is that they may play the same role in organizing oceanic flows as do invariant manifolds in simpler flows. The effective manifolds in course of their life undergo stretching and folding at progressively small scales and intersect each other in the homoclinic points in the vicinity of which fluid particles move chaotically. Trajectories of initially close fluid particles diverge rapidly in these regions, and particles from other regions appear there. It is the mechanism for effective transport and mixing of water masses in the ocean. Moreover, stable and unstable effective manifolds constitute Lagrangian transport barriers between different regions because they are material invariant curves that cannot be crossed by purely advective processes.

Motion in any preselected region in a circulation basin may be considered as a scattering problem in 
the sense that fluid particles come into the region from outside and leave it sooner or later. Passive particles are advected by an incoming flow into a mixing region, where their motion may be chaotic, and then most of them are washed away from that region. It is known in theory of chaotic scattering that there exists an abstract chaotic invariant set in a bounded region of phase space consisting of an infinite number of hyperbolic particle trajectories that never leave the mixing region (Ott, 2002; Tel et al., 2005; Budvansky et al., 2004a b). If a particle belongs to the set at an initial moment, then it remains in the mixing region forever (in theory). Most of particles sooner or later leave the mixing region, but their behavior is strongly influenced by the presence of the chaotic invariant set. Each trajectory in the set and therefore, the whole set possesses stable and unstable manifolds. Theoretically, these manifolds have infinite spatial extent, and the tracer, belonging to the stable manifold, is advected by the incoming flow into the mixing region and remains there forever. The corresponding initial conditions make up a set of zero measure. However, the particles that are initially close to those in the stable manifold follow them for a long time, eventually deviate from them and leave the mixing region along the unstable manifold. In Appendix A, we illustrate these abstract mathematical concepts with the simple model of the flow with a fixed point eddy embedded in a background steady flow with the periodic tidal component (Budvansky et al., 2004a b) and explain how they can be used to characterize inhomogeneous mixing in realistic eddy flows.

\section{Numerical Japan sea circulation model}

The Japan Sea is a deep marginal sea with shallow straits connected with the East China Sea, Okhotsk Sea and North Pacific. The Japan Sea has three deep basins, named as Tsushima and Yamato Basins in the southern sea area and the deepest and largest Japan Basin in the northern sea area. The paper is focused on simulation of the mesoscale dynamical processes over the shelf and continental slope of the Japan Basin situated in the northwestern area of the Japan Sea. The typical large scale circulation over the Northwestern Japan Sea includes, two cyclonic gyres, the cold Primorskoye Current streamed southwestward along the continental slope of the Japan Basin and the warm northern current along the slope of Japanese
Islands. The sea domain in numerical experiments is characterized by thin shelf along the continental coast of the Russian North Primorye Region (Primorsky Krai), wide shelf of the Peter the Great Bay in the South Primorye Region and steep continental slope in the whole sea area adjacent to the northwest Japan Sea coast. The southwestern cyclonic gyre over southern and central areas of the Japan Basin is simulated in the model domain as a large scale circulation.

The MHI ocean circulation model developed by N.B. Shapiro and E.N. Mikhaylova at the Marine Hydrophysical Institute, Sevastopol, Ukraine Shapiro, 2000) is a set of 3D primitive equations under the hydrostatic and Boussinesq approaches in $\mathrm{Z}$ coordinate system with a free surface boundary condition. It belongs to a class of layered models, in which the sea consists of a number of quasi-isopycnal layers. Interfacial surfaces between layers can freely move up and down and layers can deform, physically vanish (outcrop) and restore. The MHI model has been applied in Refs. (Ponomarev and Trusenkova, 2000; Trusenkova et al., 2005) for simulation of the Japan Sea large scale circulation, as well as to simulate mesoscale dynamics in the northwestern Japan Sea area adjacent to the Primorsky Krai coast. Equations of the MHI model, vertically integrated within layers, are formulated at the beta-plane, with the $x$-axis directed from the west to east and the $y$-axis directed from the south to north. A specific feature of the MHI model is that the density (buoyancy) of any layer is allowed to vary with space and time.

The present study is focused on simulation of mesoscale dynamics over the continental slope and shelf in the closed sea area of the cyclonic gyre occupying the southern and central area of the Japan Basin. The sea domain is $39^{\circ} \mathrm{N}-44^{\circ} \mathrm{N}, 129^{\circ} \mathrm{E}-$ $138^{\circ} \mathrm{E}$ with the horizontal grid steps $2.5 \mathrm{~km}$ along latitude and $2.5 \mathrm{~km}$ along longitude. The total number of the grid points is $210 \times 280$. We set 9 quasi-isopycnal layers including the upper mixed one. The bottom topography is adopted from navigation maps. Islands can not be resolved in coastline for the chosen mesh but they are represented in topography. The area of the cyclonic gyre is suggested to be closed, and we use no slip boundary conditions for current velocity at the sea domain contour including sea coast, northern, eastern, and southern boundaries.

The initial conditions for realistic summer isopycnal interfaces, temperature and salinity distribution 


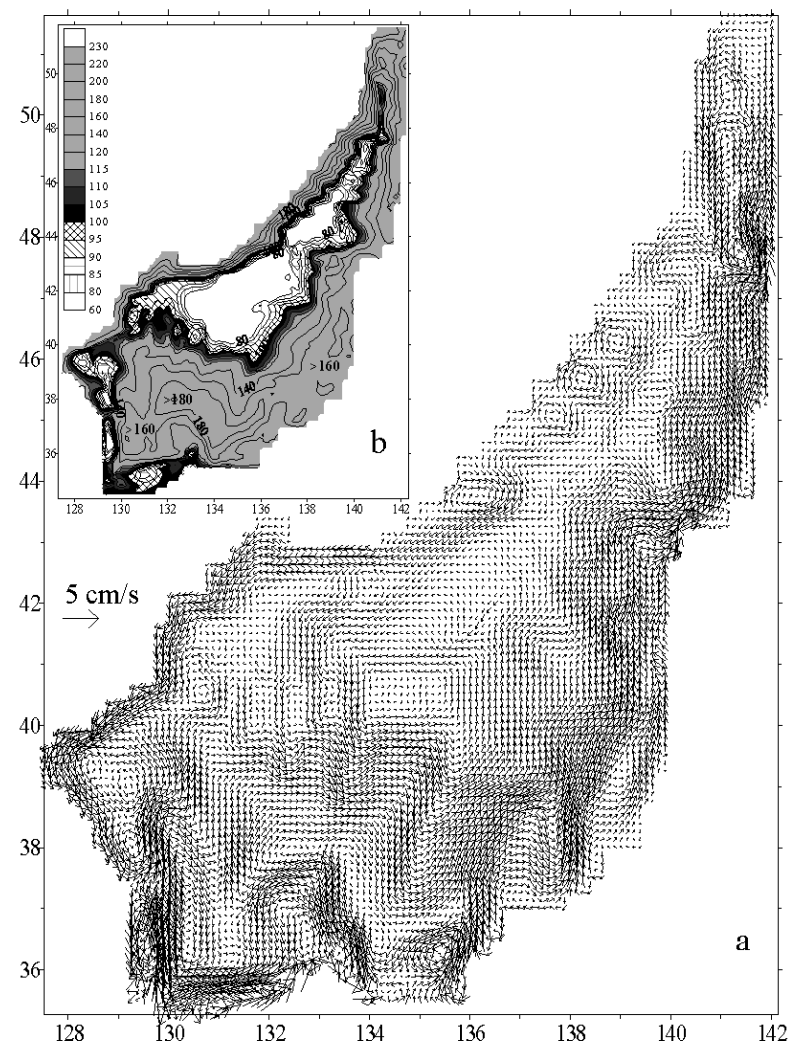

Figure 1: (a) The velocity field in the whole basin of the Japan Sea in the 6th layer of the MHI model with the horizontal resolution $1 / 16^{\circ}$ and with initial temperature and salinity distributions estimated from the CTD data of the oceanographic observations in summer 1999 (Talley et al., 2001). (b) Depths of the main pycnocline (the interface between the 5th and 6th layers of the model in August).

in the model layers have been taken from oceanographic survey 1999 (Tallev et al., 2001). The MHI model has been integrated with the time step of 4 min for a one year. The coefficients of quasiisopycnal biharmonic viscosity, harmonic viscosity, and diffusion used in the momentum and heat/salt transfer equations have been varied correspondingly from $10^{17} \mathrm{~m}^{4} / \mathrm{s}, 10^{7} \mathrm{~m}^{2} / \mathrm{s}$ and $0.4 \times 10^{7} \mathrm{~m}^{2} / \mathrm{s}$ in the model spin up (60 days) to $10^{16} \mathrm{~m}^{4} / \mathrm{s}, 10^{6} \mathrm{~m}^{2} / \mathrm{s}$ and $0.4 \times 10^{6} \mathrm{~m}^{2} / \mathrm{s}$ during other months of the warm period of a year until mid November. During the winter convection, the coefficients increase like in the spin up process. The quasi-isopycnal harmonic viscosity is applied only near the domain boundary in a warm period of a year and in the whole area in winter.

We simulate the nonlinear mesoscale eddy dy- namics over the shelf, continental slope, and Japan Basin taking into account realistic bottom topography and daily mean external atmospheric forcing. The near-surface daily atmospheric conditions have been set from the NCEP/NCAR Reanalysis. It includes short wave radiation flux, wind stress, wind speed, air temperature and precipitation.

The numerical experiments with minimized coefficients of the horizontal and vertical viscosity show the intensive mesoscale dynamics, particularly, mesoscale variability of anticyclonic/cyclonic eddies and streamers over the shelf and continental slope. The anticyclonic eddies, generated over the shelf break and continental slope, move usually southwestward along the slope like the topographic Kelvin waves with prevailing phase velocity of about $6-8 \mathrm{~cm} / \mathrm{s}$. The spatial scale of the anticyclonic eddies increases usually near the Peter the Great Bay shelf where it exceeds significantly the baroclinic Rossby deformation radius.

The current system and mesoscale dynamics over the continental slope and the Peter the Great Bay shelf change substantially from summer to winter. The strong northeastward boundary jet current is formed near the western coast of the Peter the Great Bay from late October to November when the monsoon is already changed from the summer type to the winter one. We have simulated current velocity fields in the surface mixed layer for the warm period with daily resolution in time. This warm period is associated with strong baroclinic eddy activity in the Northwestern Japan Sea.

Figure 11 demonstrates the velocity field in the whole basin of the Japan Sea in the 6th layer of the model (Ponomarev and Trusenkova, 2000; Trusenkova et al., 2005) with the horizontal resolution $1 / 16^{\circ}$ and with initial temperature and salinity distributions estimated from the CTD data of the oceanographic observations in summer 1999 (Talley et al., 2001). An annual run of atmospheric conditions corresponds to the end of the 20th century. The mesoscale eddies are generated due to the baroclinic instability in the main pycnocline and manifest themselves in the upper layers as well. The velocity field in Fig. 1]demonstrates clearly the mesoscale eddies along the coast of the Prymorsky Krai region between $43^{\circ} \mathrm{N}$ and $46^{\circ} \mathrm{N}$. 


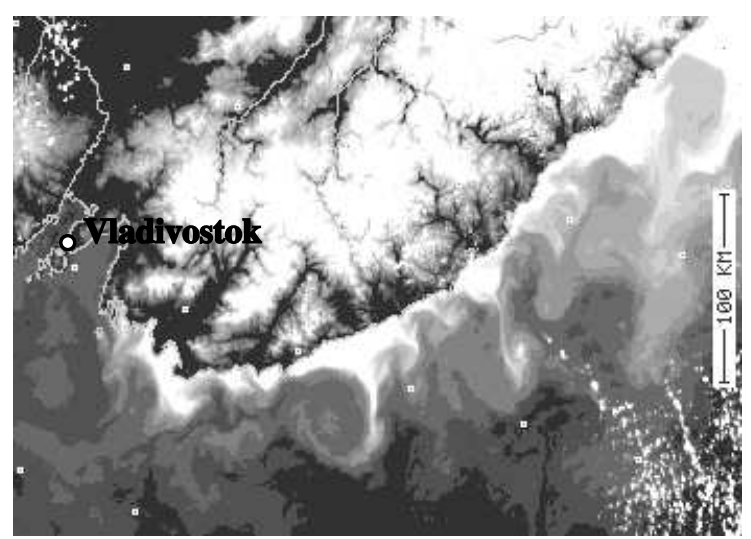

Figure 2: Satellite image of the water surface temperature in the selected region of the Japan Sea in the infrared range (NOAA AVHRR data, 15. 09. 1997). Dark and white colors correspond to low and high temperatures, respectively.

\section{Lagrangian results}

\subsection{Finite-time Lyapunov exponents and repelling material lines}

In our analysis we focus on the region between latitudes $41^{\circ} \mathrm{N}$ and $44^{\circ} \mathrm{N}$ and between longitudes $130^{\circ} \mathrm{E}$ and $136^{\circ} \mathrm{E}$ comprising the Primorskoye Current flowing to the southwest along the continental slope of the Primorsky Krai (Russia). Satellite data demonstrate anticyclonic mesoscale eddies of relatively small scale which are observed in this area directly over the steep continental slope. The satellite image of the surface temperature in the infrared range in the part of this region is shown in Fig. 2. Dark and white colors in the figure correspond to low and high temperatures, respectively. The street of the anticyclonic eddies with the scale of $50 \mathrm{~km}$ is visible along the coast of Primorsky Krai over the strip shelf and steep continental slope of the Japan Basin. Centers of the eddies are situated directly over the shelf break, $200 \mathrm{~m}$ depth. They often form pairs of strongly interacting eddies visible at sea-surface temperature satellite images. We demonstrate in Fig. 3 such vortex pairs in the NOAA AVHRR Landsat-5 (TM) and Landsat7 (ETM+) infrared images in two different years with the resolution of 120 and $60 \mathrm{~m}$, respectively (http://glovis.usgs.gov/). Each image shows the pair of two interconnected spirals with a stagnation point between them. The cores of the eddies are at the distance $25 \div 30 \mathrm{~km}$ from the coast of the Primorsky Krai. The images also show the upwelling phenomena prevailing near the coast and
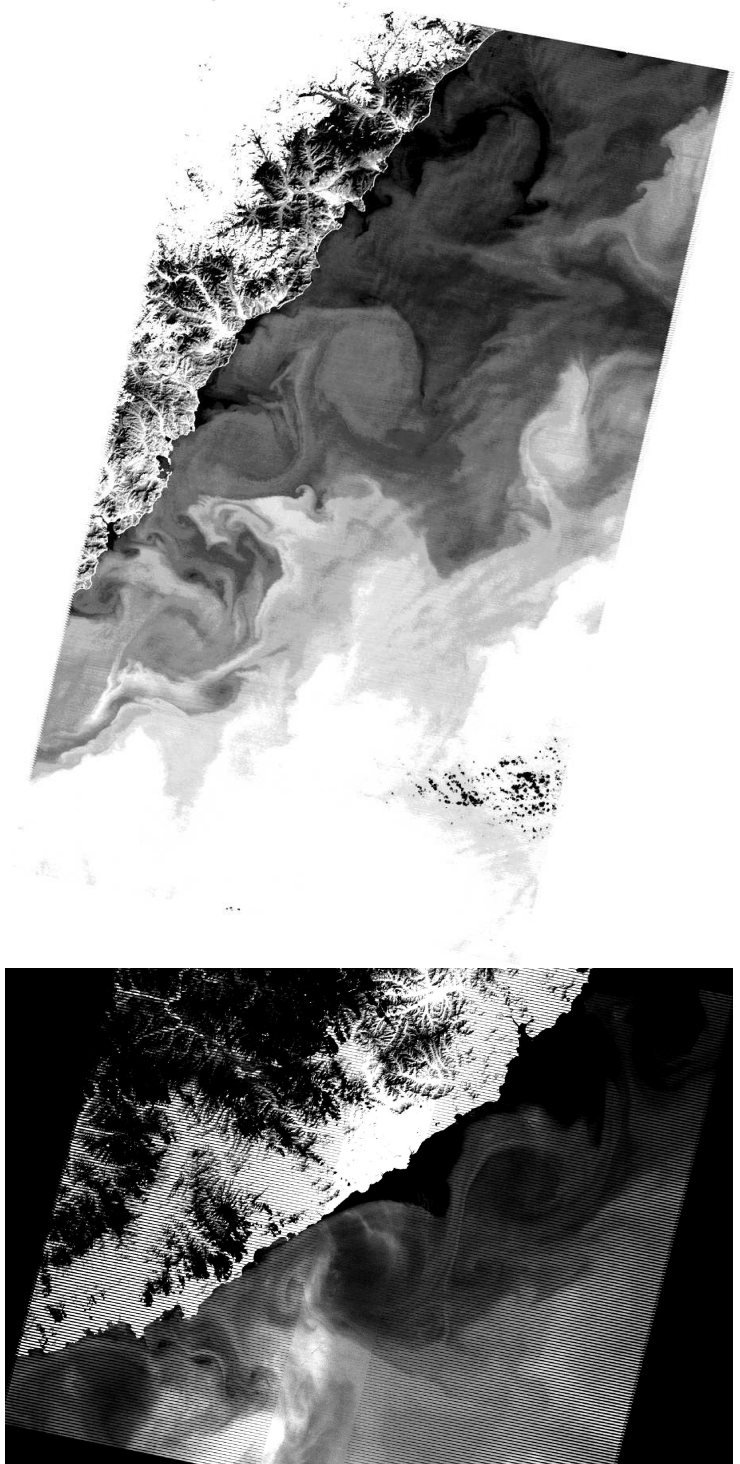

Figure 3: The NOAA AVHRR infrared images of the vortex pairs in the mesoscale eddy street associated with the Primorskoye Current. The upper panel: Landsat-5 (TM) data (29. 09. 2007) with the resolution of $120 \mathrm{~m}$. Coordinates: $43^{\circ} 38^{\prime} \mathrm{N}-45^{\circ} 35^{\prime} \mathrm{N}, 135^{\circ} 10^{\prime} \mathrm{E}-138^{\circ} 14^{\prime} \mathrm{E}$. The lower panel: Landsat-7 (ETM+) data (14.09. 2008) with the resolution of $60 \mathrm{~m}$. Coordinates: $42^{\circ} 10^{\prime} \mathrm{N}-44^{\circ} 10^{\prime} \mathrm{N}, 133^{\circ} 7^{\prime} \mathrm{E}-$ $136^{\circ} 10^{\prime} \mathrm{E}$.

cold/warm streamers injecting the cold/warm water from the coastal upwelling/offshore areas into the anticyclonic eddies. We will study in this section such a vortex pair by the Lagrangian methods using upper-layer current velocity fields from numerical experiments with the nonstationary hydro- 
dynamic MHI sea circulation model.

The mesoscale dynamics in the Primorskoye Current over the shelf and steep continental slope, where the centers of the anticyclonic eddies are situated directly over the strip shelf break, is associated with the effect of coastal Kelvin waves propagating southwestward along the shelf break. The integration of both the MHI model and diagnostic model (Fyman and Ponomarev, 2008), using observed temperature and salinity profiles from oceanographic R/V surveys, shows the anticyclonic eddies with the scale of $50 \mathrm{~km}$ and the centers over the shelf break moving downstream along the shelf and continental slope. The anticyclonic eddies of the similar scale are simulated in the wide shelf of the Peter the Great Bay. A snapshot of the vorticity field, rot $\mathbf{v}$, on one of the days in the first month of integration of the MHI model (Fig. 4a) demonstrates the complex pattern of mixing in that region with a number of anticyclonic eddies of different sizes with negative vorticity.

In theory of dynamical systems, the Lyapunov exponents, $\lambda$ 's, are known to be quantitative criteria of chaotic motion in the asymptotic limit. In practice, one computes Lyapunov exponents for a finite time. The finite-time Lyapunov exponent (FTLE) is the finite-time average of the maximal separation rate for a pair of neighbouring advected particles. The FTLE at position $\mathbf{r}$ at time $\tau$ is given by

$$
\lambda(\mathbf{r}(t)) \equiv \frac{1}{\tau} \ln \sigma(G(t)),
$$

where $\tau$ is an integration time, and $\sigma(G(t))$ denotes the largest singular value of the evolution matrix $G(t)$ which governs evolution of small displacements in linearized advection equations (see Appendix B for the derivation of formula (44)).

The FTLE is not an instantaneous separation rate, but rather measures the integrated separation between trajectories. In real oceanic flows, instantaneous streamlines can quickly diverge from actual particle's trajectories. The FTLEs adequately describe actual transport and mixing in the ocean because they are derived directly from particle's trajectories. They are especially useful in oceanography because they are mathematical analogues of drifter launching in the ocean and characterize quantitatively dispersion of water masses. Computing the FTLE field in a selected geographic region, we get the map that contains information about mixing properties in the region for a given period of time. Comparing the maps in different seasons, we get an information about variability in the region. Moreover, the Lyapunov maps enable to reveal Lagrangian coherent structures hidden in the velocity field including stable and unstable manifolds of finite-time hyperbolic trajectories, largescale transport barriers and eddies. It is interesting that the FTLE is, in fact, an Eulerian quantity, but in the same time it is a Lagrangian one because it is derived from particle's trajectories.

A uniform grid of $1000 \times 1000$ particles is advected by the numerically generated velocity field. After 50 days (starting on September, 15), the FTLEs are computed using Eq. (4). Spatial distribution of the FTLEs, plotted against initial positions in Fig. 4b, may be called a Lyapunov synoptic map. This map shows that there is a large range of positive $\lambda$ values up to 0.3 days $^{-1}$ which corresponds to Lyapunov mixing times (e-folding times) down to 3 days. We would like to stress that the plot in Fig. 4b shows values of $\lambda$ against initial particle's positions accumulated for a rather long time, 50 days.

The Lyapunov synoptic map in Fig. 4b reveals a number of structures. There are mesoscale anticyclonic eddies, forming the street along the continental slope of the Primorsky Krai, which is easily visible on the Lyapunov map and on the vorticity map (Fig. 4a). The eddy's cores are characterized by low values of the Lyapunov exponents. The particles inside the cores tend to stay therein for a comparatively long time. There are filaments that wind up around the eddy's centers in spirals which reveal transport pathways of an ejection of water.

Moreover, there are very long filaments, ridges of $\lambda$, corresponding to the largest Lyapunov exponents, which are not associated with any eddies. The ridges with largest values of $\lambda$, sandwiched between the regions with smaller $\lambda$ 's values, mean that fluid particles placed initially on one of those ridges will experience in the future strong hyperbolic behavior, i. e., they will diverge from each other at an exponential rate for the computed period of time. The ridges reveal stretching directions of the velocity field. In the language of dynamical systems theory, they approximate stable manifolds in a selected area.

To demonstrate inhomogeneity of mixing in the region under consideration, we compute the evolution of three fluid patches. The patch 3 was chosen at the ridge of the Lyapunov map (it is the left rectangular among the three ones marked in Fig. 4b) with the centroid located initially at 


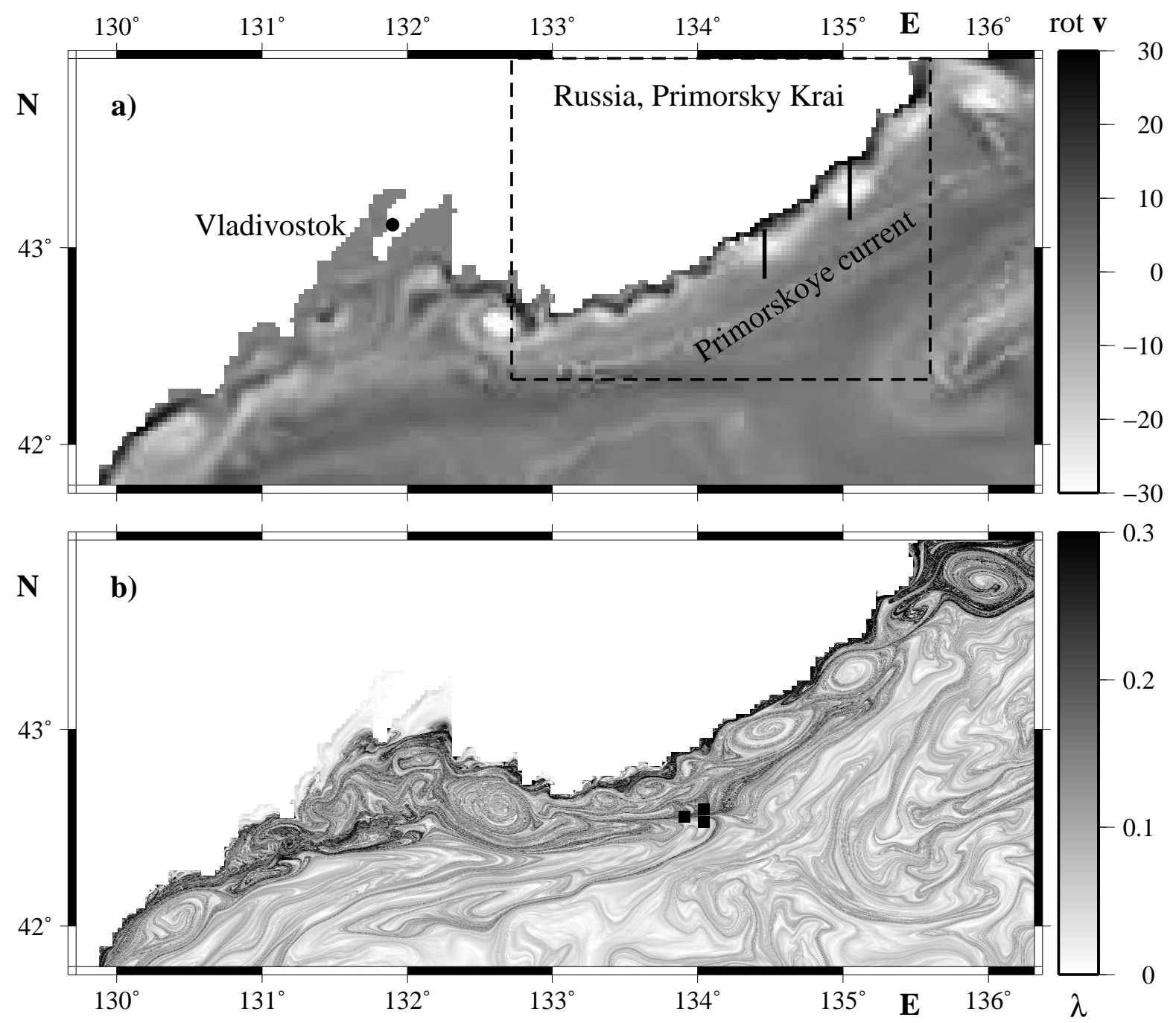

Figure 4: (a) Snapshot of the vorticity field plotted vs initial particle's positions. Color modulates the values of the vorticity rot v. (b) Lyapunov synoptic map shows the maximal FTLE, $\lambda$, vs initial particle's position. $\lambda$ is in units days ${ }^{-1}$. Integration time is 50 days.

$\left(x_{0}=133^{\circ} 54^{\prime} \mathrm{E}\right.$ and $\left.y_{0}=42^{\circ} 34^{\prime} \mathrm{N}\right)$, whereas the patches $1\left(x_{0}=134^{\circ} 2^{\prime} \mathrm{E}\right.$ and $\left.y_{0}=42^{\circ} 26^{\prime} \mathrm{N}\right)$ and $2\left(x_{0}=134^{\circ} 2^{\prime} \mathrm{E}\right.$ and $\left.y_{0}=42^{\circ} 36^{\prime} \mathrm{N}\right)$ were chosen nearby on the both sides of the ridge. Figure [5 compares their evolution for 18 days. The patches 1 and 2 remain coherent for this time but their fate is different: the patch 1 travels to the south-west, whereas the patch 2 hits the coast to the north from its initial location. Any ridge with largest values of $\lambda$ serves, in fact, as a transport barrier for waters on both its sides.

The behavior of the patch 3 in Fig. 5 is different. It undergoes strong stretching and folding to be elongated for 18 days over more than $600 \mathrm{~km}$, almost two order of magnitude greater than the patches 1 and 2 do. This is because the patch 3 was initialized at the ridge of the Lyapunov map which approximately corresponds to a stable manifold. As with any patch placed near a stable manifold, the particles inside it align along the associated unstable manifold in course of time. It takes 5-6 days for the patch 3 to reach the unstable manifold. After that time, the patch undergoes rapid stretching and folding. 


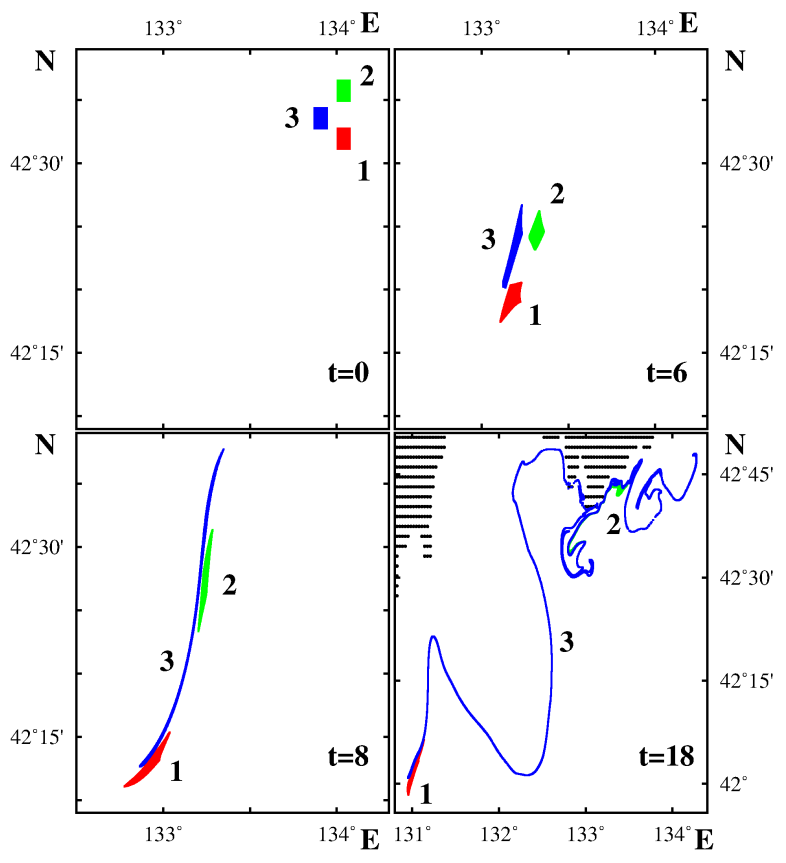

Figure 5: Evolution of the coherent patches 1 (red) and 2 (green) and the patch 3 (blue), which is strongly deformed, for 18 days. Spatial scale in the panel at $t=18$ is different from the other ones. The size of each patch is $6 \mathrm{~km}$ along the latitude and $3 \mathrm{~km}$ along the longitude with $250 \times 250$ particles in each one.

\subsection{Lagrangian diagnostic tools for revealing eddy's structure}

In this section we focus on Lagrangian study of transport and mixing of passive particles by the pair of strongly interacting anticyclonic eddies in the vortex street associated with the Primorskoye current. The satellite images of the surface temperature (Fig. 3) often demonstrate such pairs in different years. The snapshots of the vorticity field generated by the Japan Sea MHI model (Fig. 4a) and the synoptic Lyapunov map (Fig. 4b) give the evidence of the vortex pairs in the same region. In addition to the FTLE field, we propose new Lagrangian diagnostic tools for revealing eddy's structure and eddy induced transport and mixing. To be concrete we consider the most prominent pair of eddies in this region marked by the two straight lines Fig. 4a. They are also easily visible in the Lyapunov synoptic map in Fig. $4 \mathrm{~b}$.

It is worth to recall that the FTLE is an integrated quantity characterizing the divergency of nearby advected particles for a comparatively long period of time, 50 days in our case. So, the FTLE synoptic map is a field of this quantity in geographic coordinates which are initial positions of the passive particles. Figure $6 \mathrm{~b}$ is a zoom of the Lyapunov synoptic map in Fig. 4b clearly demonstrating the complex pattern of mixing of passive particles by the vortex pair selected. The values of $\lambda$ form a spiral-like structure for each of the eddies in the vortex pair. The very pair is surrounded by the ridges of the largest values of the FTLE revealing stretching directions of the velocity field which are stable manifolds of the hyperbolic trajectories of the vortex pair. The spiral-like structure, in turn, is the pair of spiral bands, one with largest values of $\lambda$ and the other one with small FTLE values. The band with largest FTLE values is a collection of initial particle positions which leave the corresponding eddy region in course of time, whereas the band with minimal FTLE values marks those particles that do not quit the eddy for 50 days. Thus, the shadowed spirals in Fig. 6b provide the Lagrangian information on the transport pathways along which advected particles quit the corresponding eddy in course of time. Using the velocity fields and the Lagrangian eddy patterns we can estimate the general speed of the anticyclonic eddies moving southwestward along the shelf break as equal to $2 \div 3 \mathrm{~cm} / \mathrm{s}$, while the current velocity in the upper mixed layer and on the sea surface is about $10 \div 20 \mathrm{~cm} / \mathrm{s}$. It corresponds to the mean speed of the Primorskoye Current in the upper layer of $200 \div 400 \mathrm{~m}$ in the main pycnocline.

The FTLE map has a fine grained structure with a number of details. To get more smoothed picture of mixing it is sometimes useful to compute the so-called exit-time map. We distribute initially $10^{6}$ particles in the box shown in Fig. 4 a and compute how long it takes for a particle with given initial position to leave the box. The corresponding map, which is more contrast than the Lyapunov one, is shown in Fig. 6a. It clearly demonstrates that the northern eddy has a prominent central homogeneous spot (the eddy's core) with large values of the exit times. The southern eddy is represented by the spiral beginning in the eddy's core.

There is the quantity that may provide a representative picture of eddy-induced advection, the number of times particles wind around the eddy's center. It is difficult to compute that number exactly for a large number of particles but it may be approximated by the number of times a given particle changes the sign of its zonal $\left(n_{x}\right)$ and meridional $\left(n_{y}\right)$ velocities unless it reaches one of the borders of 
the box shown in Fig. 近. We plot in Figs. 66 and d geographic maps of those quantities where they are coded by the color. Both the maps demonstrate clearly the spiral-like structure of the vortex pair.

To illustrate Lagrangian motion of particles in the vortex pair and compare that motion in each of the eddies, we track the evolution of material lines crossing the cores of the eddies. We take two

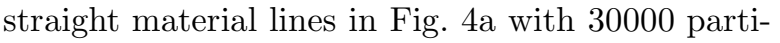
cles in each one (the same lines are shown in Fig. [6a) one of which crosses the core of the northern eddy and the other - the core of the southern eddy. Their evolution for 14 days is shown in Fig. 7 The dotted fragments in this figure appear because of insufficiently large initial density of points. The particles in the middle fragments of each line begin to rotate anticyclonically (the panel at $t=2$ ), form quickly the vortex pair (the panel at $t=6$ ) and move downstream along with the eddy's cores winding around their centers. The outer fragments of the lines elongate to the north and south along the unstable manifolds of the eddy street (the panel at $t=10$ ). At $t=14$, the northern eddy catches the southern one up and then both the eddies move together.

To give a detailed description of the structure of each eddy in the vortex pair we apply the method of particle's scattering elaborated in Ref. (Budyansky et al., 2004a). This method is explained briefly in Appendix A of the present paper. We take the same lines as shown in Figs. 4h but compute now dependencies of time of particle's exit off the selected box, $T$, the number of times particles change the sign of their meridional velocity, $n_{y}$, and the maximal FTLE on initial particle's latitude $y_{0}$ (see Figs. 8 8 , b and c, respectively).

The left column in Fig. 8 a with the latitudes $y_{0}<43^{\circ} 07^{\prime} \mathrm{N}$ represents the data for the southern eddy and the right column with the latitudes $y_{0}>43^{\circ} 07^{\prime} \mathrm{N}$ - the northern eddy. Their comparison allows to see the difference between the two eddies in the vortex pair. It is evident from Fig. 8 a that particles prefer to quit the southern eddy, including its core, more or less periodically by portions. Each portion is represented by a $U$ like segment of the $T\left(y_{0}\right)$ function which consists of a large number of particles with approximately the same time of exit and the same number of changes of the sign of their meridional velocity before leaving the selected box. It is seen in the plot $n_{y}\left(y_{0}\right)$ in Fig. $8 \mathrm{~b}$ that the particles belonging to a given $\cup$-like segment have the same values of $n_{y}$. In difference

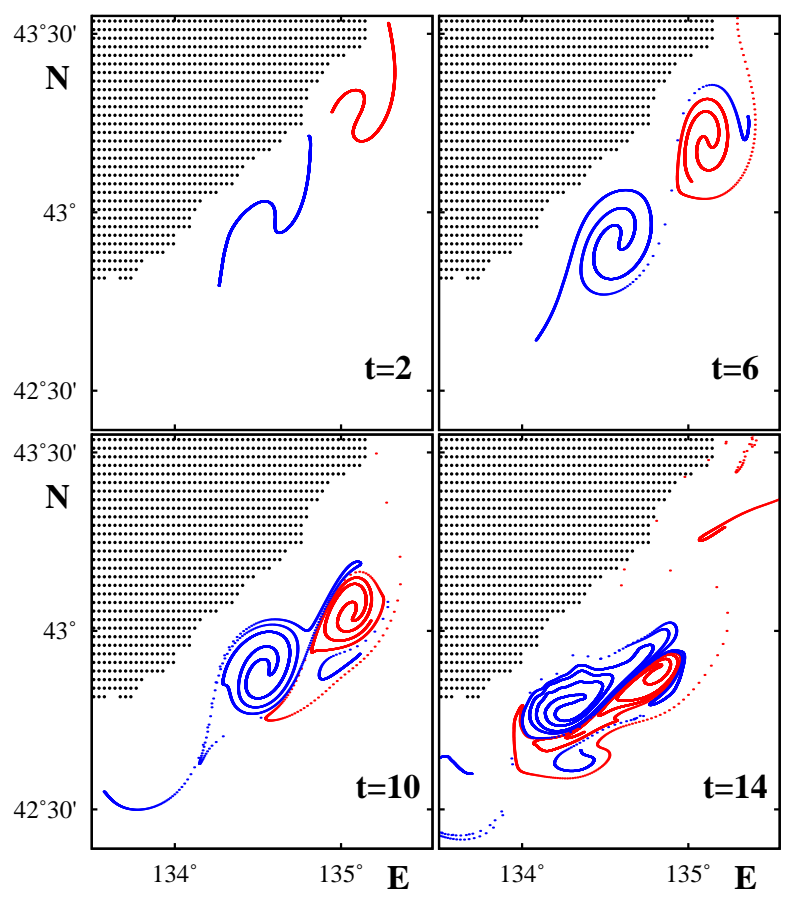

Figure 7: Evolution of the two material lines (Fig. 4 a) crossing the cores of the northern and southern eddies in the vortex pair. The red (blue) color refers to the northern (southern) eddy.

from the southern eddy, particles quit the northern eddy's core practically at the same time. In other words, the particles quit the southern eddy by portions along spiral-like transport pathways, whereas the periphery of the northern eddy exchanges water with the surrounding but its core moves coherently as a whole for a long time.

Comparison of the left and right plots $n_{y}\left(y_{0}\right)$ in Fig. 8b gives an additional information on particle advection by the two eddies. The particles in the core of the northern eddy change the sign of their meridional velocity $4 \div 8$ times before leaving the selected box (the right column), whereas the core of the southern eddy is more inhomogeneous (the left column). It is confirmed by comparing the plots $\lambda\left(y_{0}\right)$ for both the eddies in Fig. 8 ;.

The scattering plots enable to identify in the most unambiguous manner the eddy's core and its periphery. As an example let us consider the plots for the northern eddy shown in the right column in Fig. 8. The eddy's core is represented in the $T\left(y_{0}\right)$ plot in Fig. 8 8 by the smooth segment of the length $\simeq 14 \mathrm{~km}$ surrounded by the inhomogeneous struc- 

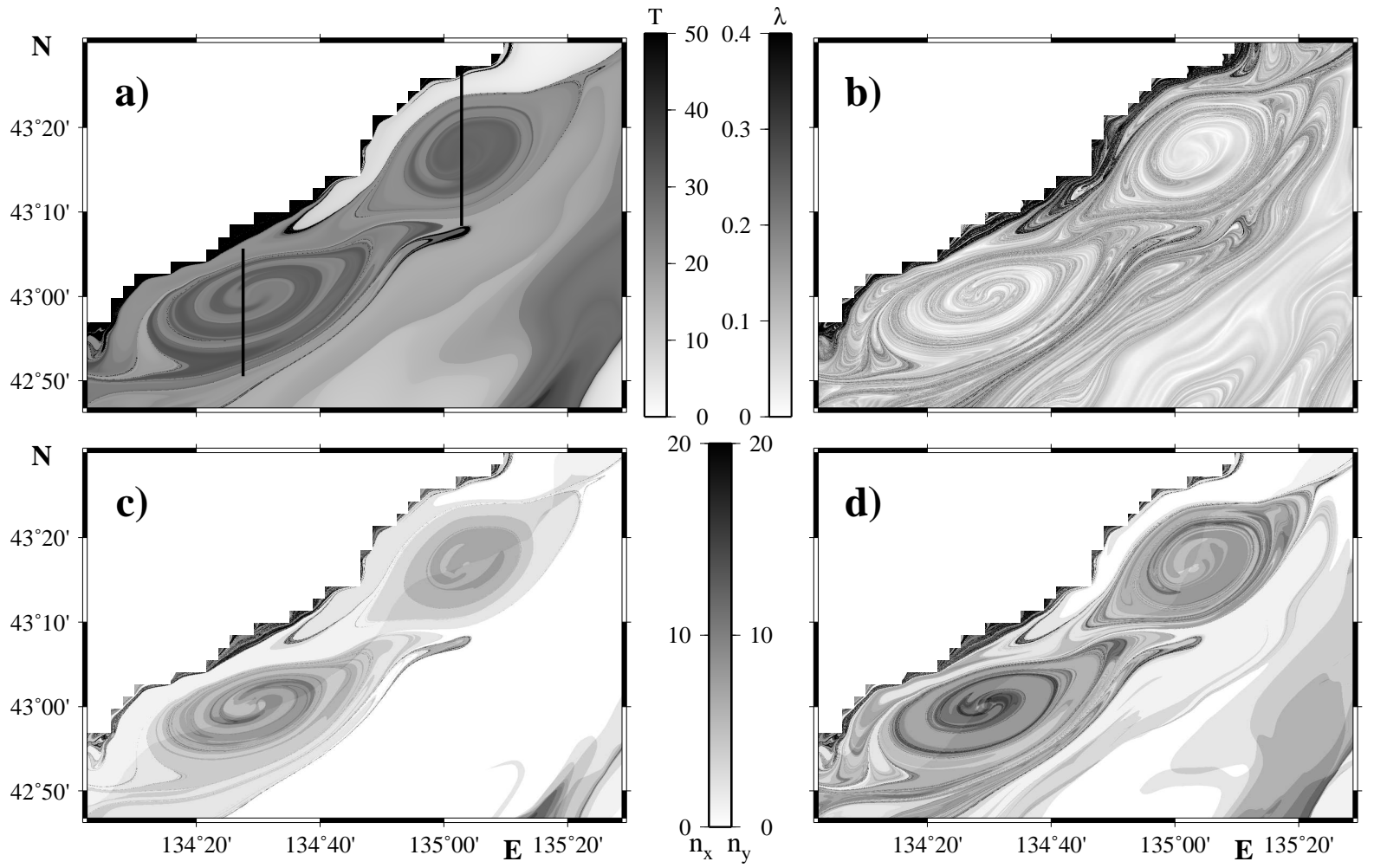

Figure 6: (a) Exit-time map shows how long in days $(T)$ it takes for a particle with a given initial position to quit the box shown in Fig. 4h. (b) Lyapunov synoptic map of the region shows the values of $\lambda$ vs initial particle's position. (c) and (d) Maps of the number of times advected particles change the sign of their zonal and meridional velocities, $n_{x}$ and $n_{y}$, respectively, unless they quit the box.

tures which should be attributed to the eddy's periphery. The $\cup$-like segments of the function $T\left(y_{0}\right)$ beyond the eddy's core represent the water masses evolving more or less coherently and quitting the eddy by portions. This process is manifested in Fig. 6 as spirals of the northern eddy which are less prominent than those for the southern one. The motion of particles in the eddy's periphery is erratic due to numerous intersections of effective stable and unstable manifolds.

Figure $8 \mathrm{~b}$ gives the picture of circulation on submesoscales. We show in Appendix A by means of the example with a simple vortex-current system that both the trapping time for particles in the mixing zone and the number of their full turns around the vortex have a hierarchical fractal structure as functions of initial particle's position. Both these functions (see Figs. A.10a and b) are singular on a Cantor set of initial conditions. Due to periodicity of that idealized flow it became possible to explain in detail transport and mixing of passive particles.
Numerically generated velocity fields in the ocean are, of course, aperiodic. Moreover, we deal with moving eddies which are patches of nonzero vorticity. So, we could not expect the simplified picture of scattering of material lines by a moving eddy resembling that is shown in Appendix A. Nevertheless, we find a kind of resemblance of the plots in Figs. $8 \mathrm{a}$ and $\mathrm{b}$ with the corresponding plots in Figs. A.10a and b. The function $T\left(y_{0}\right)$ is a graph with smooth U-like segments intermitted with badly resolved ones. There is no, of course, singularities at the ends of smooth segments because of lacking of periodicity of the velocity field and transient character of effective stable and unstable manifolds. The plot $n_{y}\left(y_{0}\right)$ in Fig. $8 \mathrm{~b}$ is a hierarchy of epistrophes, but it is organized in a much more complicated way as compared to the ones in Refs. (Budyansky et al., 2004a) due to the same reasons. There is no evident fractal structure in both the plots but there is a kind of self-similarity and strong difference in behavior of neighbour particles whose exit times $T$ 

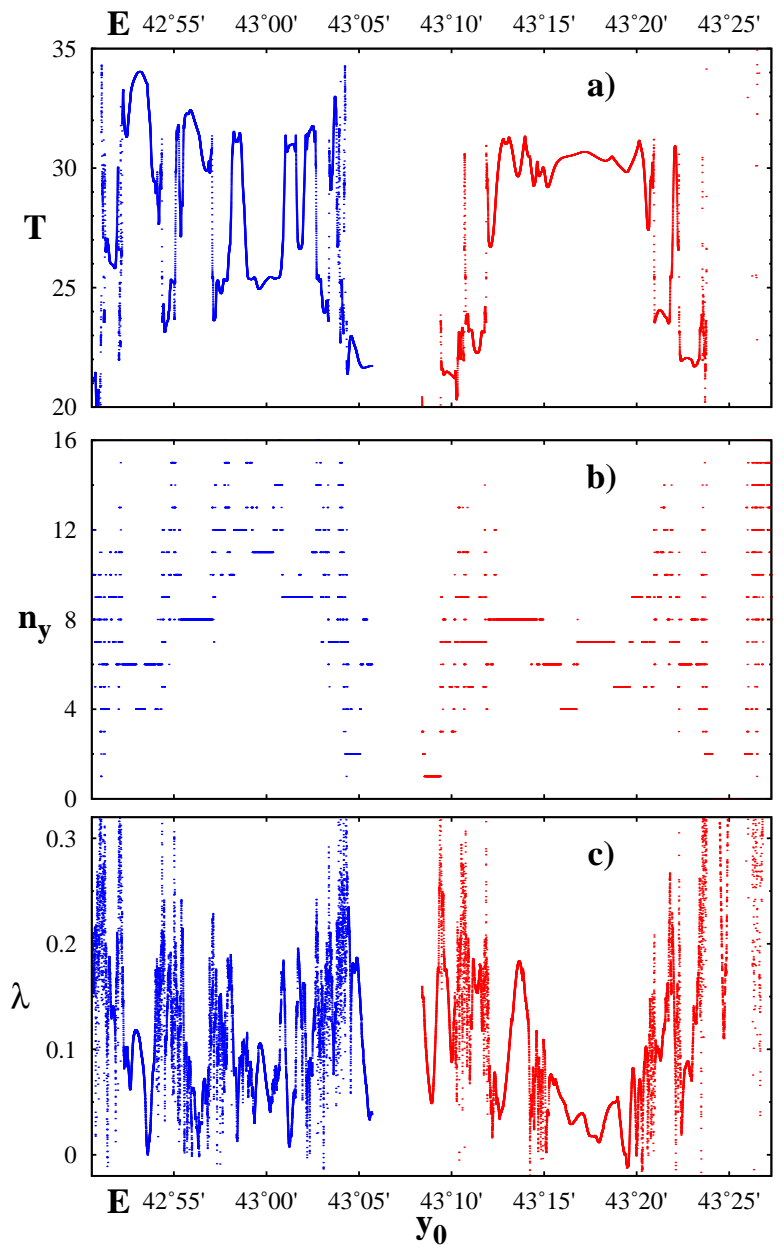

Figure 8: Evolution of the same two material lines as in Fig. 7 The left (blue) and right (red) columns refer to the southern and northern eddies, respectively. (a) Time of exit off the box $T$ in days vs initial particle's latitude position $y_{0}$. (b) Number of times, $n_{y}$, the particles change the sign of their meridional velocity before they leave the box vs $y_{0}$. (c) The corresponding maximal finite-time Lyapunov exponent vs $y_{0}$.

and the number $n_{y}$ may differ by an order of magnitude for a rather short period of time. Particles in each smooth segment in Fig. 8k have approximately the same number of changes of the velocity sign. So, each such segment represents a coherent portion of the whole material line which is washed away off the selected box simultaneously. The segments in Fig. 8a, which are not smooth, consist of a number of portions with their own fate and very different values of $T$ and $n_{y}$.

Important oceanographic information can be gained by computing so-called streaklines in the ac-

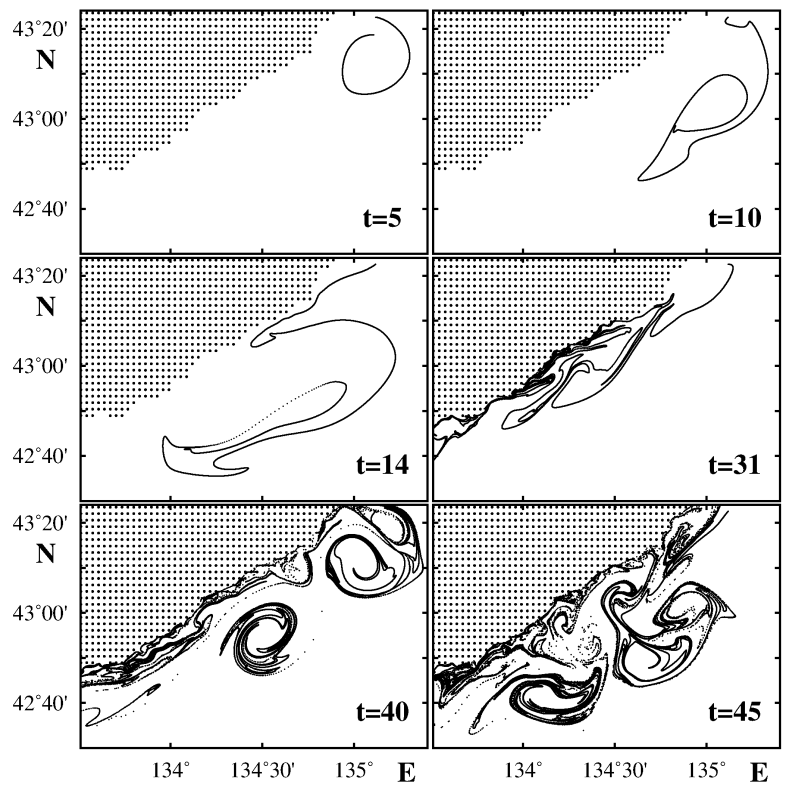

Figure 9: Snapshots of the streakline obtained by injection of a dye for 45 days. The injection point $\left(x_{0}=135^{\circ} 7^{\prime} \mathrm{E}\right.$, $y_{0}=43^{\circ} 23^{\prime} \mathrm{N}$ ) was chosen at the periphery of one of the eddies present there at the initialization time.

tive regions of the flow. Streakline at the time moment $t$, passing through a point $(x, y)$, is a curve composed of all the fluid particles which passed through that point before the moment $t$. Injecting a dye into a point on the flow plane, one can visualize the corresponding streakline. Recalling the recent catastrophic oil spill at the bottom in the Gulf of Mexico, it is evident that tracking the evolution of streaklines in numerically generated or measured velocity fields may provide useful information about possible oil transport.

Figure 9 illustrates complex form of the streakline in the region under consideration. The injection point $\left(x_{0}=135^{\circ} 7^{\prime} \mathrm{E}, y_{0}=43^{\circ} 23^{\prime} \mathrm{N}\right)$ was chosen to be at the periphery of one of the anticyclonic eddies present there at the start of the injection. That's why the injected particles begin to encircle the eddy anticyclonically (see the panels on 5th and 10th days). The initial portion of the injected particles moves downstream together with that eddy (the panel at $t=14$ ). The dotted fragments of the streakline appear because of insufficiently large initial density of points. The particles, released during the phase without eddies near the injection point, begin to move downstream (the panels at $t=10$ and $t=14$ ). Moving with the Primorskoye Current, 
they successfully catch up the downstream eddies, and the streakline begins to draw up all the anticyclonic eddies present in the region (see the panels at $t=31, t=40$ and $t=45$ ). The resulting streakline at $t=45$ gives an approximate image of the effective unstable manifold of the whole region.

\section{Conclusion}

We have demonstrated in this paper that Lagrangian tools and methods of dynamical systems theory can help to gain new information on surface transport and mixing on both mesoscales and submesoscales in the ocean. We have focused on the selected region of the Japan Sea comprising the coastal Primorskoye Current with a street of anticyclonic mesoscale eddies. Computing Lagrangian trajectories for a large number of particles advected by the MHI numerical model, we have studied eddyinduced surface transport and mixing in that region. We have developed the method to compute the FTLEs for any velocity field and plotted the Lyapunov synoptic map with a high resolution which can be used to quantify mixing processes. The Landsat satellite infrared images, high resolution numerical experiments with the MHI circulation model and Lagrangian modelling of the mesoscale and submesoscale dynamics in the Primorskoye Current system have shown strongly interacting mesoscale anticyclonic eddies generated over the shelf break and steep continental slope in the northwestern area of the Japan Sea.

The main attention has been paid to Lagrangian study of transport and mixing by a vortex pair of strongly interacting eddies which often occur in that region in summer and autumn periods. We proposed new Lagrangian diagnostic tools, the time of exit of particles off a selected box, the number of changes of the sign of zonal and meridional velocities, and computed synoptic maps for these quantities. Along with the Lyapunov map, they have been shown to be able to reveal mesoscale eddies, meso- and submesoscale filaments, repelling material lines, hyperbolic and non-hyperbolic regions in the sea. In particular, we have found that the eddies have a prominent spiral-like structure resembling the spiral patterns at satellite images in that region.

Based on the theory of chaotic scattering, we developed the technique to track evolution of clusters of particles, streaklines and material lines and applied it to study in detail transport and mixing in- duced by the vortex pair. The so-called scattering functions, dependencies of the exit times and the number of times particles wind around the eddy's center on initial particle positions, give us important oceanographic information on eddy's structure and eddy-induced transport and mixing. In particular, they allow to identify in the most unambiguous manner the eddy's cores and periphery and to discover that the eddies release the water to the surrounding by portions.

Lagrangian approach to transport and mixing problems seems to be perspective because the results of computation of finite-time and finite-scale Lyapunov exponents, exit times and winding numbers, tracking the evolution of streaklines, material lines and patches of fluid particles will be more and more realistic along with improvement of high-resolution numerical models of the ocean circulation. The numerical results obtained within that approach can be readily compared to the results of surface-drifter and subsurface-float experiments which are rapidly becoming a common experimental technique in oceanography (Garraffo et al., 2001; Molcard et al., 2003; Özgökmen et al., 2003; Poje et al., 2002; Molcard et al., 2006). This approach can be used as well for making predictions about possible oil and other pollutant transport, biological productivity and other applications. An interesting application of Lagrangian approach to study marine ecosystem dynamics has been found recently in Ref. (Tew Kai et al., 2009) where it has been demonstrated that frigatebirds may trace precisely Lagrangian coherent structures in the Mozambique Channel which are ridges in the field of the finite-size Lyapunov exponent.

\section{Acknowledgments}

We would like to thank V. Dubina for providing us the satellite images in Fig. 3 and anonymous reviewers for valuable comments. The work was supported partially by the Program "Fundamental Problems of Nonlinear Dynamics" of the Russian Academy of Sciences, by the Russian Foundation for Basic Research (project no. 09-05-98520) and by the Prezidium of the Far-Eastern Branch of the RAS. 


\section{Appendix A. Geometry of chaotic scatter- ing of particles in a simple vortex model}

It is instructive to demonstrate typical underlying structures that govern complicated mixing and transport by ocean eddies and introduce some special geometric concepts with an oversimplifed model of the oceanic flow with a topographic eddy embedded in a background steady flow with the periodic tidal component (Budyansky et al., 2004a , b) .

Passive particles, advected by a steady flow with the periodic component directed along the $y$-axis, enter the mixing region where a fixed point eddy with the singular point at $x=y=0$ is located and then wash out to an outflow region. Let us put a large number of particles outside the mixing region on a line segment, crossing the current, with the fixed value of their $y_{0}$-coordinates and different values of $x_{0} i$. We compute the time $T$, when the particles reach the line $y=6$, and the number of particle's rotations $n$ around the point eddy before reaching this line. It is a problem of chaotic scattering (Ott, 2002).
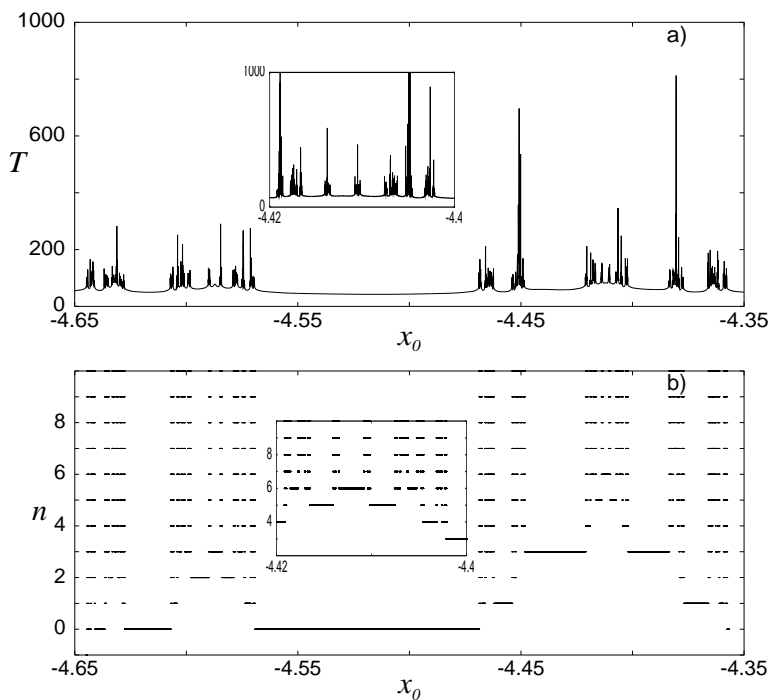

Figure A.10: (a) Fractal dependence of the trapping time $T$ on initial particle's positions $x_{0}$ with the inset showing a 20fold magnification of one of the singularity zones. (b) Number of particle's rotations $n$ around the point eddy before reaching a fixed line. Mechanism of generating the fractal with magnification of a small segment corresponding to the inset in the panel (a).

Figure A.10 demonstrates the typical scattering function $T\left(x_{0}\right)$ with an uncountable number of sin- gularities which are unresolved in principle. The inset in Fig. A.10 shows a zoom by the factor of 20 of one of these singularity zones. Successive magnifications confirm a self-similarity of the function with increasing values of the trapping times $T$. To give an insight into a mechanism of generating the fractal, we plot in Fig. A.10 segments of the initial string with a large number of particles which are trapped in the mixing region after $n$ rotations around the fixed point eddy. After each rotation, a portion of particles is washed out in the downstream region with $y \geq 6$. This process resembles the mechanism of generating the famous Cantor set but is more complicated. It is well known (see, for example, (Ott, 2002)) that in constructing the middle third Cantor set one takes the closed interval $[0,1]$ and removes open middle third intervals from each of the intervals remaining at each stage of the process. It is seen from Fig. A.10 that, starting with the given interval of initial points, different portions of tracers are washed out by the flow from the intervals remaining after each rotation around the vortex. Continuing in this way ad infinitum, we get a Cantor set of remaining initial points.

Due to periodicity of that idealized flow it became possible to explain in detail transport and mixing of passive particles (Budyansky et al., 2004a). The point is that there exists an invariant chaotic set (saddle set) $\Lambda$ (Ott, 2002; Tel et al., 2005). This set is defined as the set of all trajectories (except for Kolmogorov-Arnold-Moser tori and cantori) that never leave the mixing region. It consists of an infinite number of unstable periodic and aperiodic (chaotic) trajectories. The particles belonging to $\Lambda$ remain on it forever. However, their measure is zero.

Each trajectory, belonging to $\Lambda$, and therefore the whole set has stable, $\Lambda_{s}$, and unstable, $\Lambda_{u}$, manifolds which are infinite material curves. The stable manifold of the chaotic set is defined as the invariant set of trajectories approaching those in $\Lambda$ as $t \rightarrow \infty$. The unstable manifold $\Lambda_{u}$ is defined as the stable manifold corresponding to time-reversed dynamics. Following trajectories in $\Lambda_{s}$, particles, advected by the incoming flow, enter the mixing region and remain there forever. Particles that are initially close to those in $\Lambda_{s}$ follow the corresponding trajectories, then deviate from them and eventually leave the mixing region along the unstable manifold $\Lambda_{u}$. So, if one chooses a material line in the incoming flow, crossing the stable manifold $\Lambda_{s}$, it is expected that some advected particles will stay 
in the mixing zone forever giving rise to singularities in the $T\left(y_{0}\right)$ function. It means that this function has a self-similar (fractal) structure with smooth $\cup$-like segments with singularities at their ends intermitted with wildly oscillating fragments with are unresolvable under magnification in principle.

Particles, which are chosen to be close to $\Lambda_{s}$ in the incoming flow, enter along $\Lambda_{s}$ into the mixing zone where they remain for a long time and eventually exit the region along the unstable manifold. The set of segments with equal number of turns around the vortex, $n$, were called "epistrophes" in Ref. (Budyansky et al., 2004a) following to Ref. (Mitchell et al., 2003). These epistrophes were shown to make up a hierarchy (Fig. A.10b). Each epistrophe converges to a limit point on the corresponding material-line segment. The endpoints of each segment of the $n$ th-level epistrophe are the limit points of an $n+1$ th-level epistrophe. The lengths of segments in an epistrophe decreases in geometric progression. The common ratio of all the progressions $q$ is related to the maximal Lyapunov exponent for the saddle points as follows: $\lambda=-\ln q / 2 \pi$. The hierarchy of epistrophes in Fig. A.10b determines transport of particles, and its fractal properties are generated by the infinite sequences of intersections of the advected material line with stable and unstable manifolds of the chaotic invariant set $\Lambda$. The similar fractal-like picture of chaotic transport and mixing has been found in more realistic models for a periodically meandering jet current like the Gulf Stream and the Kuroshio (Koshel and Prants, 2006; Prants et al., 2006; Uleysky et al., 2007).

\section{Appendix B. Finite-time Lyapunov expo- nents}

In this Appendix we derive the formula (44) for the finite-time Lyapunov exponents (FTLE) used in this paper to compute the Lyapunov synoptic map for the Japan sea region selected (Fig. 4b). The general problem of particle's advection by a flow in an abstract $n$-dimensional space is described by the $n$-dimensional system of nonlinear ordinary differential equations in the vector form

$$
\begin{gathered}
\dot{\mathbf{x}}=\mathbf{f}(\mathbf{x}, t), \quad \mathbf{x}=\left(x_{1}, \ldots, x_{n}\right), \\
\mathbf{f}(\mathbf{x}, t)=\left(f_{1}\left(x_{1}, \ldots, x_{n}, t\right), \ldots, f_{n}\left(x_{1}, \ldots, x_{n}, t\right)\right) .
\end{gathered}
$$

The Lyapunov exponent at an arbitrary point $\mathbf{x}_{\mathbf{0}}$ is given by

$$
\Lambda\left(\mathbf{x}_{\mathbf{0}}\right)=\lim _{t \rightarrow \infty} \lim _{\|\delta \mathbf{x}(0)\| \rightarrow 0} \frac{\ln (\|\delta \mathbf{x}(t)\| /\|\delta \mathbf{x}(0)\|)}{t},
$$

where $\delta \mathbf{x}(t)=\mathbf{x}_{\mathbf{1}}(t)-\mathbf{x}_{\mathbf{0}}(t), \mathbf{x}_{\mathbf{0}}(t)$ and $\mathbf{x}_{\mathbf{1}}(t)$ are solutions of the set (B.1), $\mathbf{x}_{\mathbf{0}}(0)=\mathbf{x}_{\mathbf{0}}$. The limit exists, is the same for almost all the choices of $\delta \mathbf{x}(0)$ and has a clear geometrical sense: trajectories of two nearby particles diverge in time exponentially (in average) with the exponent given by the Lyapunov exponent.

Due to smallness of $\delta \mathbf{x}$ one can linearize the set (B.1) in a vicinity of some trajectory $\mathbf{x}_{\mathbf{0}}(t)$ and obtain the system of time-dependent linear equations (Greene and Kim, 1987)

$$
\left(\begin{array}{c}
\delta \dot{x}_{1} \\
\cdots \\
\delta \dot{x}_{n}
\end{array}\right)=J(t)\left(\begin{array}{c}
\delta x_{1} \\
\cdots \\
\delta x_{n}
\end{array}\right)
$$

where $J(t)$ is the Jacobian matrix of the system (B.1) along the trajectory $\mathbf{x}_{\mathbf{0}}(t)$

$$
J(t)=\left(\begin{array}{lll}
\frac{\partial f_{1}\left(\mathbf{x}_{\mathbf{0}}(t), t\right)}{\partial x_{1}} & \ldots & \frac{\partial f_{1}\left(\mathbf{x}_{\mathbf{0}}(t), t\right)}{\partial x_{n}} \\
\ldots \ldots \ldots \ldots \ldots \ldots \ldots \ldots \ldots \ldots \ldots \ldots \ldots \\
\frac{\partial f_{n}\left(\mathbf{x}_{\mathbf{0}}(t), t\right)}{\partial x_{1}} & \ldots & \frac{\partial f_{n}\left(\mathbf{x}_{\mathbf{0}}(t), t\right)}{\partial x_{n}}
\end{array}\right) .
$$

Solution of the linear system B.3 can be found with the help of the evolution matrix $G\left(t, t_{0}\right)$

$$
\left(\begin{array}{c}
\delta x_{1}(t) \\
\ldots \ldots \\
\delta x_{n}(t)
\end{array}\right)=G\left(t, t_{0}\right)\left(\begin{array}{c}
\delta x_{1}\left(t_{0}\right) \\
\ldots \ldots \\
\delta x_{n}\left(t_{0}\right)
\end{array}\right)
$$

The evolution matrix obeys the differential equation which can be obtained after substituting (B.5) into B.3

$$
\dot{G}=J G,
$$

with the initial condition $G\left(t_{0}, t_{0}\right)=I$, where $I$ is the unit matrix. Any evolution matrix has the important property

$$
G\left(t, t_{0}\right)=G\left(t, t_{1}\right) G\left(t_{1}, t_{0}\right) .
$$

One can write the singular-value decomposition of the evolution matrix as follows:

$$
G\left(t, t_{0}\right)=U\left(t, t_{0}\right) D\left(t, t_{0}\right) V^{T}\left(t, t_{0}\right),
$$

where $U, V$ are orthogonal and $D=$ $\operatorname{diag}\left(\sigma_{1}, \ldots, \sigma_{n}\right)$ is diagonal. The quantities 
$\sigma_{1}, \ldots, \sigma_{n}$ are called singular values of the matrix $G$. The Lyapunov exponents are defined via singular values of the evolution matrix as follows:

$$
\Lambda_{i}=\lim _{t \rightarrow \infty} \frac{\ln \sigma_{i}\left(t, t_{0}\right)}{t-t_{0}}
$$

Quantities

$$
\lambda_{i}\left(t, t_{0}\right)=\frac{\ln \sigma_{i}\left(t, t_{0}\right)}{t-t_{0}}
$$

are called finite-time Lyapunov exponents (Okushima, 2003). Thus, the FTLE is the ratio of the logarithm of the maximal possible stretching in a given direction to a time interval $t-t_{0}$.

Let us consider now our specific problem of particle's advection on a plane with $2 \times 2$ evolution matrix and the singular-value decomposition

$$
\begin{array}{r}
G=U D V^{T} \Rightarrow\left(\begin{array}{ll}
a & b \\
c & d
\end{array}\right)=\left(\begin{array}{cc}
\cos \phi_{2} & -\sin \phi_{2} \\
\sin \phi_{2} & \cos \phi_{2}
\end{array}\right) \times \\
\left(\begin{array}{cc}
\sigma_{1} & 0 \\
0 & \sigma_{2}
\end{array}\right)\left(\begin{array}{cc}
\cos \phi_{1} & -\sin \phi_{1} \\
\sin \phi_{1} & \cos \phi_{1}
\end{array}\right) \cdot \quad(\mathrm{B} .10)
\end{array}
$$

Solution of these four algebraic equations has the form

$$
\begin{aligned}
\sigma_{1} & =\frac{\sqrt{(a+d)^{2}+(c-b)^{2}}+\sqrt{(a-d)^{2}+(b+c)^{2}}}{2}, \\
\sigma_{2} & =\frac{\sqrt{(a+d)^{2}+(c-b)^{2}}-\sqrt{(a-d)^{2}+(b+c)^{2}}}{2}, \\
\phi_{1} & =\frac{\arctan 2(c-b, a+d)-\arctan 2(c+b, a-d)}{2}, \\
\phi_{2} & =\frac{\arctan 2(c-b, a+d)+\arctan 2(c+b, a-d)}{2},
\end{aligned}
$$

where function $\arctan 2$ is defined as

$$
\arctan 2(y, x)= \begin{cases}\arctan (y / x), & x \geq 0 \\ \arctan (y / x)+\pi, & x<0\end{cases}
$$

Equation (B.6) can not be numerically integrated over a large time because the elements of the corresponding evolution matrix grow exponentially if one of the Lyapunov exponents is positive. However, we can divide a large time interval on subintervals with the duration which is less or order of the Lyapunov time, $t_{\lambda}=1 / \lambda$, and represent the whole evolution matrix as a product of evolution matrices computed on these subintervals using the property (B.7). We compute this product and the corresponding singular values using the GNU Multiple Precision Arithmetic Library (http://gmplib.org) in order to preserve the absolute precision of our representation of the evolution matrix.

\section{References}

Abraham, E.R., Bowen, M.M., 2002. Chaotic stirring by a mesoscale surface-ocean flow. Chaos: An Interdisciplinary Journal of Nonlinear Science 12, 373-381.

Aref, H., 1984. Stirring by chaotic advection. Journal of Fluid Mechanics 143, 1-21.

Artale, V., Boffetta, G., Celani, A., Cencini, M., Vulpiani, A., 1997. Dispersion of passive tracers in closed basins: Beyond the diffusion coefficient. Physics of Fluids 9, 31623171

Beron-Vera, F.J., Olascoaga, M.J., Goni, G.J., 2008. Oceanic mesoscale eddies as revealed by Lagrangian coherent structures. Geophys. Res. Lett. 35, L12603.

Budyansky, M., Uleysky, M., Prants, S., 2004a. Chaotic scattering, transport, and fractals in a simple hydrodynamic flow. Journal of Experimental and Theoretical Physics 99, 1018-1027.

Budyansky, M., Uleysky, M., Prants, S., 2004b. Hamiltonian fractals and chaotic scattering of passive particles by a topographical vortex and an alternating current. Physica D: Nonlinear Phenomena 195, 369-378.

d'Ovidio, F., Fernández, V., Hernández-García, E., López, C., 2004. Mixing structures in the Mediterranean Sea from finite-size Lyapunov exponents. Geophys. Res. Lett. 31, L17203.

d'Ovidio, F., Isern-Fontanet, J., López, C., HernándezGarcía, E., García-Ladona, E., 2009. Comparison between Eulerian diagnostics and finite-size Lyapunov exponents computed from altimetry in the Algerian basin. Deep Sea Research Part I: Oceanographic Research Papers 56, 1531.

Fyman, P., Ponomarev, V., 2008. Diagnostic simulation of sea currents in the Peter the Great Bay based on FERHRI oceanographic surveys. Pacific Oceanography 4, 56-64.

García-Olivares, A., Isern-Fontanet, J., García-Ladona, E., 2007. Dispersion of passive tracers and finite-scale Lyapunov exponents in the Western Mediterranean Sea. Deep Sea Research Part I: Oceanographic Research Papers 54, $253-268$.

Garraffo, Z.D., Mariano, A.J., Griffa, A., Veneziani, C., Chassignet, E.P., 2001. Lagrangian data in a highresolution numerical simulation of the North Atlantic: I. Comparison with in situ drifter data. Journal of Marine Systems 29, 157-176.

Gildor, H., Fredj, E., Steinbuck, J., Monismith, S., 2009. Evidence for submesoscale barriers to horizontal mixing in the ocean from current measurements and aerial photographs. Journal of Physical Oceanography 39, 19751983.

Greene, J.M., Kim, J.S., 1987. The calculation of Lyapunov spectra. Physica D: Nonlinear Phenomena 24, 213-225.

Haller, G., 2000. Finding finite-time invariant manifolds in two-dimensional velocity fields. Chaos: An Interdisciplinary Journal of Nonlinear Science 10, 99-108.

Haller, G., Poje, A.C., 1998. Finite time transport in ape- 
riodic flows. Physica D: Nonlinear Phenomena 119, 352380.

Haller, G., Yuan, G., 2000. Lagrangian coherent structures and mixing in two-dimensional turbulence. Physica D: Nonlinear Phenomena 147, 352-370.

Ide, K., Small, D., Wiggins, S., 2002. Distinguished hyperbolic trajectories in time-dependent fluid flows: analytical and computational approach for velocity fields defined as data sets. Nonlinear Processes in Geophysics 9, 237-263.

Izrailsky, Y., Koshel, K., Stepanov, D., 2008. Determination of optimal excitation frequency range in background flows. Chaos: An Interdisciplinary Journal of Nonlinear Science 18, 013107.

Jones, C., Winkler, S., 2002. Chapter 2. Invariant manifolds and Lagrangian dynamics in the ocean and atmosphere, in: Fiedler, B. (Ed.), Handbook of Dynamical Systems. Elsevier Science. volume 2, pp. 55-92.

Koshel, K., Sokolovskiy, M., Davies, P., 2008. Chaotic advection and nonlinear resonances in an oceanic flow above submerged obstacle. Fluid Dynamics Research 40, 695.

Koshel, K.V., Prants, S.V., 2006. Chaotic advection in the ocean. Physics Uspekhi 49, 1151-1178.

Lehahn, Y., d'Ovidio, F., Lévy, M., Heifetz, E., 2007. Stirring of the northeast Atlantic spring bloom: A Lagrangian analysis based on multisatellite data. J. Geophys. Res. 112, C08005.

Lekien, F., Coulliette, C., Mariano, A.J., Ryan, E.H., Shay, L.K., Haller, G., Marsden, J., 2005. Pollution release tied to invariant manifolds: A case study for the coast of Florida. Physica D: Nonlinear Phenomena 210, 1-20.

Lipphardt, B., Small, D., Kirwan, A., Wiggins, S., Ide, K., Grosch, C., Paduan, J., 2006. Synoptic Lagrangian maps: Application to surface transport in Monterey Bay. Journal of Marine Research 64, 221-247(27).

Mancho, A.M., Hernández-García, E., Small, D., Wiggins, S., Fernández, V., 2008. Lagrangian transport through an ocean front in the northwestern mediterranean sea. Journal of Physical Oceanography 38, 1222-1237.

Mancho, A.M., Small, D., Wiggins, S., 2006. A tutorial on dynamical systems concepts applied to Lagrangian transport in oceanic flows defined as finite time data sets: Theoretical and computational issues. Physics Reports 437, 55-124.

Miller, P.D., Jones, C.K.R.T., Rogerson, A.M., Pratt, L.J., 1997. Quantifying transport in numerically generated velocity fields. Physica D: Nonlinear Phenomena 110, 105122.

Mitchell, K.A., Handley, J.P., Tighe, B., Delos, J.B., Knudson, S.K., 2003. Geometry and topology of escape. I. Epistrophes. Chaos: An Interdisciplinary Journal of Nonlinear Science 13, 880-891.

Molcard, A., Piterbarg, L.I., Griffa, A., Özgökmen, T.M., Mariano, A.J., 2003. Assimilation of drifter observations for the reconstruction of the Eulerian circulation field. J. Geophys. Res. 108, 3056.

Molcard, A., Poje, A.C., zgkmen, T.M., 2006. Directed drifter launch strategies for Lagrangian data assimilation using hyperbolic trajectories. Ocean Modelling 12, 268289.

Okushima, T., 2003. New method for computing finite-time Lyapunov exponents. Phys. Rev. Lett. 91, 254101.

Olascoaga, M.J., Rypina, I.I., Brown, M.G., Beron-Vera, F.J., Koçak, H., Brand, L.E., Halliwell, G.R., Shay, L.K., 2006. Persistent transport barrier on the West Florida Shelf. Geophys. Res. Lett. 33, L22603.
Ott, E., 2002. Chaos in dynamical systems; 2nd ed. Cambridge University Press, Cambridge, U.K.

Ottino, J.M., 1989. The Kinematics of Mixing: Stretching, Chaos, and Transport. Cambridge University Press, Cambridge, U.K.

Özgökmen, T.M., Molcard, A., Chin, T.M., Piterbarg, L.I., Griffa, A., 2003. Assimilation of drifter observations in primitive equation models of midlatitude ocean circulation. J. Geophys. Res. 108, 3238.

Pierrehumbert, R.T., Yang, H., 1993. Global chaotic mixing on isentropic surfaces. Journal of the Atmospheric Sciences 50, 2462-2480.

Poje, A.C., Toner, M., Kirwan, A.D., Jones, C.K.R.T., 2002. Drifter launch strategies based on Lagrangian templates. Journal of Physical Oceanography 32, 1855-1869.

Ponomarev, V., Trusenkova, O., 2000. Circulation patterns of the Japan Sea. La Mer 38, 189-198.

Prants, S.V., Budyansky, M.V., Uleysky, M.Y., Zaslavsky, G.M., 2006. Chaotic mixing and transport in a meandering jet flow. Chaos: An Interdisciplinary Journal of Nonlinear Science 16, 033117.

Samelson, R.M., 1992. Fluid exchange across a meandering jet. Journal of Physical Oceanography 22, 431-444.

Samelson, R.M., Wiggins, S., 2006. Lagrangian Transport in Geophysical Jets and Waves: The Dynamical Systems Approach (Interdisciplinary Applied Mathematics). Springer.

Shadden, S.C., Lekien, F., Marsden, J.E., 2005. Definition and properties of Lagrangian coherent structures from finite-time Lyapunov exponents in two-dimensional aperiodicflows. Physica D: Nonlinear Phenomena 212, 271-304.

Shapiro, N., 2000. Formation of a circulation in the quasiisopycnic model of the Black Sea taking into account the stochastic nature of the wind stress. Physical Oceanography $10,513-531$.

Talley, L., Lobanov, V., Tishchenko, P., Ponomarev, V., Sherbinin, A., Luchin, V., 2001. Hydrographic observations in the Japan/East Sea in winter, 2000, with some results from summer, 1999, in: Danchenkov, M. (Ed.), Oceanography of the Japan Sea. Proceedings of CREAMS'2000, Dalnauka Publishing House, Vladivostok, Russia. pp. 25-32.

Tel, T., de Moura, A., Grebogi, C., Karolyi, G., 2005. Chemical and biological activity in open flows: A dynamical system approach. Physics Reports 413, 91-196.

Tew Kai, E., Rossi, V., Sudre, J., Weimerskirch, H., Lopez, C., Hernandez-Garcia, E., Marsac, F., Garçon, V., 2009. Top marine predators track lagrangian coherent structures. PNAS 106, 8245-8250.

Trusenkova, O., Ishida, H., Ratner, Y., Stanichny, S., Ponomarev, V., 2005. Numerical modeling of heat and freshwater fluxes, temperature, and salinity at the Japan Sea surface. Doboku Gakkai Ronbunshu 2005, 125-142.

Uleysky, M.Y., Budyansky, M.V., Prants, S.V., 2007. Effect of dynamical traps on chaotic transport in a meandering jet flow. Chaos: An Interdisciplinary Journal of Nonlinear Science 17, 043105.

Waugh, D.W., Abraham, E.R., Bowen, M.M., 2006. Spatial variations of stirring in the surface ocean: A case study of the Tasman Sea. Journal of Physical Oceanography 36, 526-542. 